\title{
Whole exome sequencing in ADHD trios from single and multi-incident families implicates new candidate genes and highlights polygenic transmission
}

\author{
Bashayer R. Al-Mubarak $\mathbb{D}^{1,2} \cdot$ Aisha Omar ${ }^{1} \cdot$ Batoul Baz $\mathbb{D}^{1,2} \cdot$ Basma Al-Abdulaziz $^{1,3} \cdot$ Amna I. Magrashi $^{1}$. \\ Eman Al-Yemni ${ }^{1,2} \cdot$ Amjad Jabaan $^{2} \cdot$ Dorota Monies $^{2,4} \cdot$ Mohamed Abouelhoda $^{2,4,5} \cdot$ Dejene Abebe $^{6}$. \\ Mohammad Ghaziuddin ${ }^{7}$ Nada A. Al-Tassan ${ }^{1,2}$
}

Received: 26 September 2019 / Revised: 26 February 2020 / Accepted: 10 March 2020 / Published online: 1 April 2020

(c) The Author(s) 2020. This article is published with open access

\begin{abstract}
Several types of genetic alterations occurring at numerous loci have been described in attention deficit hyperactivity disorder (ADHD). However, the role of rare single nucleotide variants (SNVs) remains under investigated. Here, we sought to identify rare SNVs with predicted deleterious effect that may contribute to ADHD risk. We chose to study ADHD families (including multi-incident) from a population with a high rate of consanguinity in which genetic risk factors tend to accumulate and therefore increasing the chance of detecting risk alleles. We employed whole exome sequencing (WES) to interrogate the entire coding region of 16 trios with ADHD. We also performed enrichment analysis on our final list of genes to identify the overrepresented biological processes. A total of 32 rare variants with predicted damaging effect were identified in 31 genes. At least two variants were detected per proband, most of which were not exclusive to the affected individuals. In addition, the majority of our candidate genes have not been previously described in ADHD including five genes (NEK4, NLE1, PSRC1, PTP4A3, and TMEM183A) that were not previously described in any human condition. Moreover, enrichment analysis highlighted brain-relevant biological themes such as "Glutamatergic synapse", "Cytoskeleton organization", and " $\mathrm{Ca}^{2+}$ pathway". In conclusion, our findings are in keeping with prior studies demonstrating the highly challenging genetic architecture of ADHD involving low penetrance, variable expressivity and locus heterogeneity.
\end{abstract}

\section{Introduction}

The World health organization (WHO) estimates (10-20\%) of children worldwide to be affected with some form of childhood-onset neuropsychiatric disorder [https://www.who.

Supplementary information The online version of this article (https:// doi.org/10.1038/s41431-020-0619-7) contains supplementary material, which is available to authorized users.

Bashayer R. Al-Mubarak

BAl-Mubarak@kfshrc.edu.sa

Nada A. Al-Tassan

naltassan@kfshrc.edu.sa

1 Behavioral Genetics unit, Department of Genetics, King Faisal Specialist Hospital and Research Center, P.O Box 3354, Riyadh 11211, Saudi Arabia

2 Saudi Human Genome Program, King Abdulaziz City for Science and Technology, Riyadh, Saudi Arabia int/mental_health/maternal-child/child_adolescent/en/]. These disorders, if left unmanaged, can impair normal functioning and development. Perhaps the most common example of such conditions is attention deficit hyperactivity disorder (ADHD), characterized by a persistent pattern of inattention and/or hyperactivity-impulsivity. In some cases, these symptoms continue into adulthood [1].

Like other neuropsychiatric disorders (e.g., autism, schizophrenia, and depression), the exact causes of ADHD

3 National center for genomics technology, King Abdulaziz City for Science and Technology, Riyadh, Saudi Arabia

4 Department of Genetics, King Faisal Specialist Hospital and Research Center, P.O Box 3354, Riyadh 11211, Saudi Arabia

5 Systems and Biomedical Engineering Department, Faculty of Engineering, Cairo University, Giza, Egypt

6 Psychiatry Department, King Faisal Specialist Hospital and Research Center, P.O Box 3354, Riyadh 11211, Saudi Arabia

7 University of Michigan, Ann Arbor, MI, USA 
are not yet clearly understood. The earliest evidence for genetic contribution to ADHD came from family, twin and adoption studies consistently demonstrating high heritability (up to $88 \%$ ) [2]. While this disorder may aggregate in families, it tends not to segregate in a typical Mendelian manner. This is true not only for ADHD, but also for most of complex disorders, whereby risk to the disorder is several folds higher in the relatives of the proband compared with the general population. Also, disease risk was found to be proportional with the degree of relatedness [3, 4]. The current consensus is that ADHD is a complex condition influenced by multiple genetic, social, and environmental factors [5].

Various genome-wide approaches have been employed to investigate the genetic basis of ADHD. For a comprehensive review see $[2,6]$. The first wave of studies applied genetic linkage methods on sibling pairs, small families, or multigenerational families in search for genetic variants with large effect. Because of the high prevalence of ADHD (possibly due to increased recognition of the disorder), later studies focused on finding common single nucleotide polymorphisms (SNPs) with modest effects that are significantly associated with the disorder in case-control samples. In these studies one of two main strategies were adopted: candidate gene association or genome-wide association. The former strategy is hypothesis-driven, whereby the selection of candidate genes is based on existing knowledge about; (1) their relevant biological roles, (2) their participation to a recognized ADHD drug target pathway, and (3) their previous association with ADHD. Results from this type of studies were often inconsistent for the same locus. However, only through genome-wide association studies (GWAS) meta-analysis that researchers were able to identify significant associations across studies [7].

Unlike candidate gene association studies, GWAS are hypothesis-free interrogation of the entire genome for significant risk loci (SNPs). Previous GWAS of ADHD failed to find variants with genome-wide significance even when they were combined in a meta-analysis. This is expected given the highly stringent $P$ value threshold $\left(5 \times 10^{-8}\right)$ required in this approach, which can only be achieved using a very large sample size [8]. Thus far, only a single study was able to detect association signal with global significance [7]. In this well-powered study (20,183 cases and 35,191 controls), Demontis et al. identified 12 independent risk loci that were also found by another group to be a robust set from which polygenic risk scores could be derived for reliable prediction of some of ADHD concurrent traits [9].

Although up to one-third (estimates ranging from 0.1 to 0.28) of total ADHD heritability is thought to be based on inherited common SNPs, a large portion of the heritable risk remains unaccounted for $[4,10]$. This so-called "missing heritability" is likely to be, at least partially, explained by rare genetic variants. The first attempts to address the role of rare variants in ADHD susceptibility were focused on studying copy number variants (CNVs). While enrichment for large CNVs ( $\geq 100 \mathrm{kbp})$ has been observed in a couple of studies [11, 12], the majority of the reported CNVs had reduced penetrance and limited replication across studies $[2,13]$. Only recently, have next generation sequencing (NGS) research begun to explore the role of another class of rare variants (single nucleotide variants (SNVs)) in ADHD. However, a very limited number of studies have been published so far [14-19]. In all of these studies exome sequencing was applied for identification of rare functional SNVs either in preselected set of genes [14-16] or across the genome in a small sample size [16-19].

Collectively around 359 genes (counts from ADHDgene database, http://adhd.psych.ac.cn/index.do [accessed on 17.12.19] [20]) have been published in ADHD genetic studies. Most of these studies were conducted in Asian or European populations. The increasing realization of the disadvantages of lack of diversity calls for new diversityincreasing strategies to minimize bias and ensure worldwide applicability of the genetic findings. Such strategies are the path for realizing the promise of personalized medicine for all. The present study contributes to the growing efforts to enrich diversity by including an underrepresented nonEuropean population. We report here the first attempt to explore the role of inherited and de novo variants (SNVs and CNVs) in ADHD (parents-child) trios of Arab descent. Using whole exome sequencing (WES), we applied our inhouse analysis pipeline to identify potential ADHD risk variants.

\section{Subjects and methods}

\section{Participants}

A total of 16 Saudi families with at least one child affected with ADHD were recruited in this study after obtaining signed informed consent in accordance with the Declaration of Helsinki and the institution's relevant committees; Institutional Review Board (IRB), Research Ethics committee and Basic Research Committee. All experimental protocols used in this study were authorized under an IRBapproved project (RAC\#2120001). Recruitment was done through the psychiatry department at King Faisal Specialist Hospital and Research Centre (KFSHRC). All of the approached families agreed to participate (no withdrawals). Male to female ratio of the enrolled cases was 3:1 and the mean age was 12 years (Table 1). Probands were assessed by a trained medical team (child psychiatrist and neurologist) and diagnosed according to the diagnostic and statistical manual of mental disorders (DSM-IV) criteria. Our 
Table 1 Age and gender distribution of enrolled ADHD cases.

\begin{tabular}{|c|c|c|c|}
\hline Family-ID & $\begin{array}{l}\text { Affected } \\
\text { individuals }\end{array}$ & Gender & $\begin{array}{l}\text { Age at recruitment } \\
\text { (years) }\end{array}$ \\
\hline \multirow[t]{4}{*}{$\mathrm{F} 1$} & $\mathrm{P}$ & M & 17 \\
\hline & AS & M & 19 \\
\hline & AS & M & 13 \\
\hline & AS & M & 7 \\
\hline $\mathrm{F} 2$ & $\mathrm{P}$ & M & 8 \\
\hline F5 & $\mathrm{P}$ & M & 11 \\
\hline \multirow[t]{2}{*}{ F7 } & $\mathrm{P}$ & M & 16 \\
\hline & AS & M & 17 \\
\hline \multirow[t]{2}{*}{ F9 } & $\mathrm{P}$ & M & 13 \\
\hline & AS & $\mathrm{F}$ & 17 \\
\hline \multirow[t]{2}{*}{ F10 } & $\mathrm{P}$ & $\mathrm{F}$ & 16 \\
\hline & AS & M & 21 \\
\hline F12 & $\mathrm{P}$ & $\mathrm{F}$ & 11 \\
\hline F14 & $\mathrm{P}$ & M & 9 \\
\hline F15 & $\mathrm{P}$ & $\mathrm{F}$ & 8 \\
\hline F16 & $\mathrm{P}$ & M & 6 \\
\hline \multirow[t]{2}{*}{ F17 } & $\mathrm{P}$ & $\mathrm{F}$ & 12 \\
\hline & AS & M & 8 \\
\hline \multirow[t]{2}{*}{ F19 } & $\mathrm{P}$ & M & 10 \\
\hline & $\mathrm{AF}$ & M & NA \\
\hline \multirow[t]{2}{*}{ F21 } & $\mathrm{P}$ & M & 10 \\
\hline & AS no DNA & $\mathrm{F}$ & NA \\
\hline \multirow[t]{4}{*}{ F22 } & $\mathrm{P}$ & M & 15 \\
\hline & AS & M & 14 \\
\hline & AS & M & 10 \\
\hline & AS & M & 5 \\
\hline F24 & $\mathrm{P}$ & M & 9 \\
\hline \multirow[t]{4}{*}{ F25 } & $\mathrm{P}$ & M & 16 \\
\hline & AS & M & 14 \\
\hline & AS & M & 13 \\
\hline & $\mathrm{AS}^{\mathrm{a}}$ & $\mathrm{F}$ & 4 \\
\hline
\end{tabular}

$P$ proband, $A F$ affected father, $A S$ affected sibling, $M$ male, $F$ female, $N A$ not available or not applicable.

${ }^{\text {a }}$ Suspected ADHD case.

exclusion criteria included ADHD secondary to syndromes with known genetic causes such as Fragile $\mathrm{X}$ syndrome, Tuberous Sclerosis, Rett syndrome, Angelman, Prader-Willi syndrome, or Phenylketonuria. Therefore, only cases with non-syndromic ADHD were selected. Blood samples for DNA extraction, were collected from all available consenting family members (parents as well as affected and unaffected siblings).

\section{Copy number variation analysis}

All recruited samples were surveyed for CNVs in genes listed in (Table S1). The list contains genes from AutismKB core dataset (with a total score $\geq 20$ ), International Multisite ADHD Genetics (IMAGE) candidate risk genes [21] and genes participating in neurotransmitter systems that were not included in the aforementioned sets. CytoScan ${ }^{\mathrm{TM}} \mathrm{HD}$ Suite (Affymetrix, Santa Clara, CA, USA) was used for genome-wide detection of CNVs. Targeted analysis of CNVs in the selected genes was performed using Chromosome Analysis Suite 3.0 (ChAS 3.0). All genomic locations were based on GRCh37/hg19 human genome assembly. Gains were defined as ( $\log 2$ ratio of copy) values greater than 0.58 and loss as values less than -1 .

\section{Whole exome sequencing and data analysis}

The exomes of 16 (parents-child) trios were captured using whole exome AmpliSeq kit and sequenced on Ion Proton ${ }^{\mathrm{TM}}$ System platforms (up to $200 \mathrm{bp}$, single reads). NGS raw data are deposited at the Saudi Human Genome Program (SHGP) repository [https://genomics.saudigenomeprogram. org/en/]. All variants described in this study have been submitted to LOVD [https://databases.lovd.nl/shared/genes] public repository. Sample processing and all three stages of data analysis (primary, secondary, and tertiary) were performed as previously described [22]. The WES workflow applied in this study is summarized in Fig. 1.

\section{Variants filtering, validation, and prioritization}

Variants obtained by applying the four possible modes of inheritance (autosomal recessive, autosomal dominant, Xlinked, and de novo) were retained if present in genes with positive brain expression in three out of four databases; GTEx [23], BioGPS [24], CGAP [25], and human brain transcriptome (HBT) [26]. Next, only functional variants (missense, small insertions/deletions (indels) and loss of function $(\mathrm{LoF})$ ) with minor allele frequency (MAF) of $<1 \%$ ) in population databases (1000 Genomes project, ExAC and Kaviar) and in the local ethnically matching Saudi Human Genome Program normal controls database (SHGPdb $>2379$ exomes) were selected for Sanger validation. The resulting variants were prioritized based on their functional effect prediction whereby only those predicted to be "deleterious" by at least two out of three bioinformatics tools (CADD, PredictSNP2 and FATHMM-MKL) [27-29] were considered. At the gene level, intolerance to variation (missense and LoF) metrics (pLI and $\mathrm{Z}$ score) were extracted for each identified gene with validated variant(s). However, although these metrics can be helpful when combined with other prediction tools, they are inadequate to infer or exclude pathogenicity when used solely [22]. In addition, publicly available variants or gene databases such as dbSNP, SFARI Gene [https://gene.sfari.org/autdb/ Welcome.do], AutismKB [http://autismkb.cbi.pku.edu.cn/], 
Fig. 1 Schematic illustration of the study design and analysis workflow. F9 trio did not pass QC check therefore; it was excluded from further analysis. Dollar symbol represents the average variant count per sample. Hat symbol represents the average number of validated prioritized variants per proband.

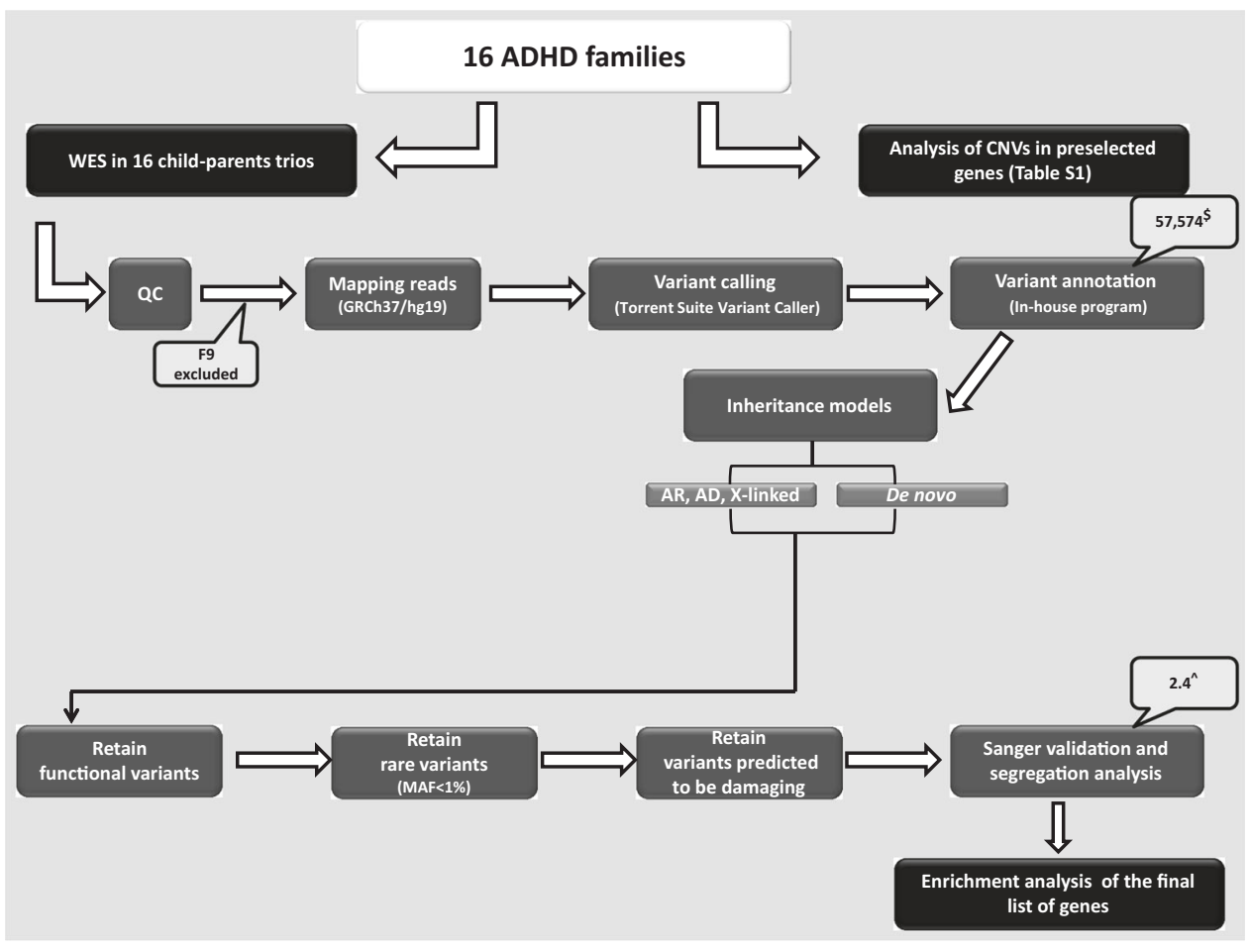

and ADHDgene [http://adhd.psych.ac.cn/] were checked for previous reports of the variants/genes identified herein. When possible, segregation analysis was performed on affected as well as unaffected siblings.

\section{Relatedness assessment}

Relationships between samples were computed using two algorithms. First we utilized VCFtools program package (with option-relatedness) to calculate the "unadjusted" $\mathrm{A}_{\mathrm{jk}}$ relatedness statistic based on Yang et al. method. In the second method, shared homozygosity was calculated by comparing the homozygous variants $(\mathrm{MAF}>1 \%$ ) between each pair of individuals and counting the number of overlapping variants after normalization. For detailed description refer to the supplementary material.

\section{Functional enrichment analysis}

Two enrichment analysis tools were employed to discover biological processes overrepresented in our gene list. The first was WEB-based Gene Set Analysis Toolkit (WebGestalt) [http://www.webgestalt.org] which serves as an integrated data mining system for functional enrichment analysis of large sets of genes. For detailed description refer to the supplementary material. In addition to WebGestalt, we utilized the Reactome Knowledgebase [www.reactome. org] which functions as an archive for biomolecular pathways besides offering overrepresentation data analysis.

\section{Results}

\section{Analysis of CNVs}

None of the genes included in our list (Table S1) were harbored in the regions with copy number alterations except for a heterozygous copy loss in SHANK3 that was identified in probands from F5 and F15. The deleted region spans the entire gene in F5, whereas in F15 it was found to overlap with parts of $S H A N K 3$. Of note, our analysis revealed CNVs in two genes that were not included in our gene list but are worth reporting here given their reported link to autism spectrum disorder (ASD) [30, 31]. The first was NOTCH1, in which copy gain was detected in probands of F10 and F17. The second was NRXN3 that was found to harbor heterozygous copy loss in F19. Locations and details of the detected CNVs are listed in Table 2

\section{WES data, performance, and statistics}

Results of relatedness assessment and demographic information are summarized in Table S2. Performance metrics of the WES runs including, number of reads per base that map to the reference genome, the percentage of target region coverage and sequencing depth are summarized in Table S3. High performance metrics were achieved indicating high quality mapping and variant calling. Type and number of the detected variants before applying the filtering pipeline are listed in Table S4. All trios passed quality 


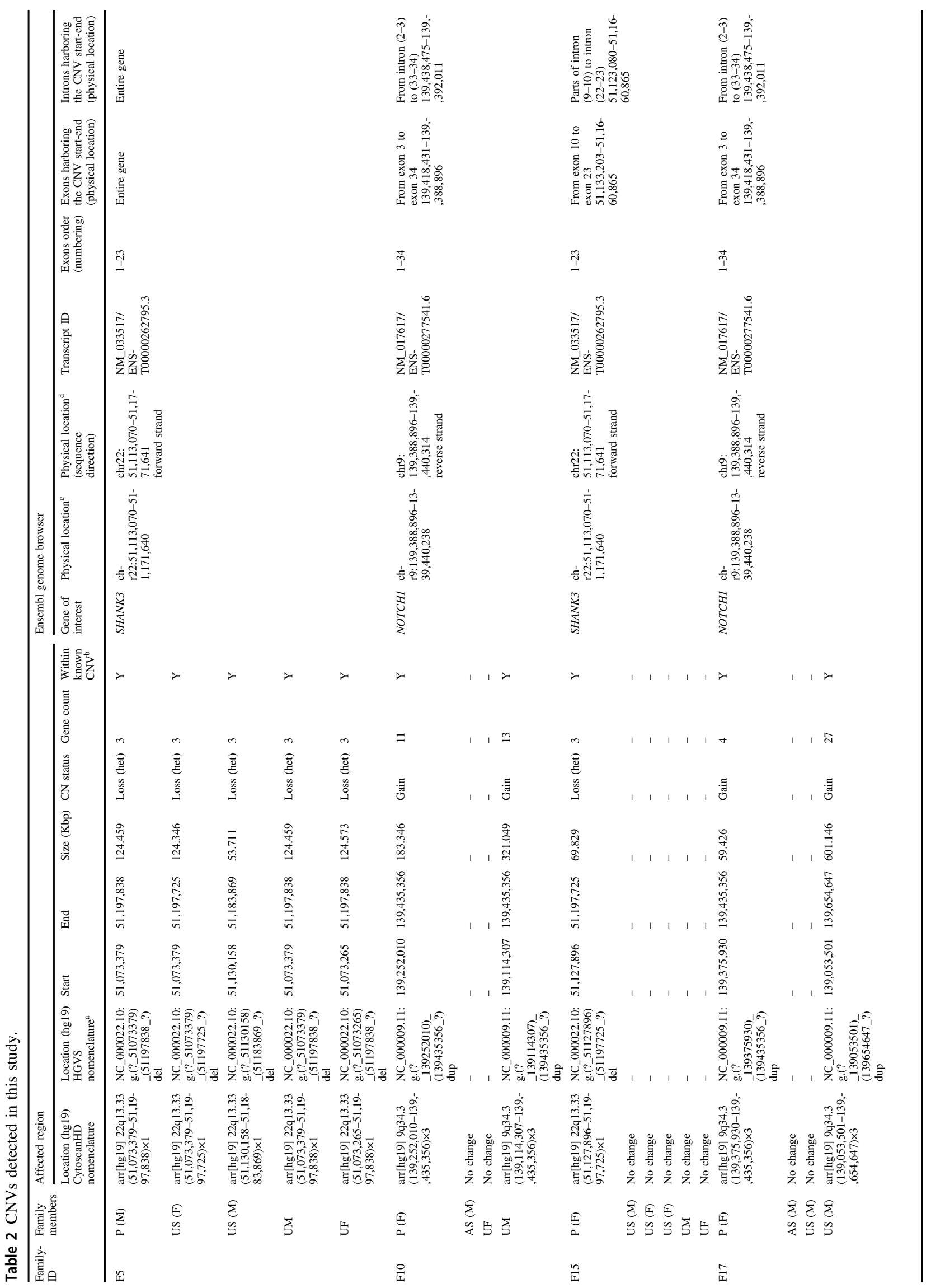




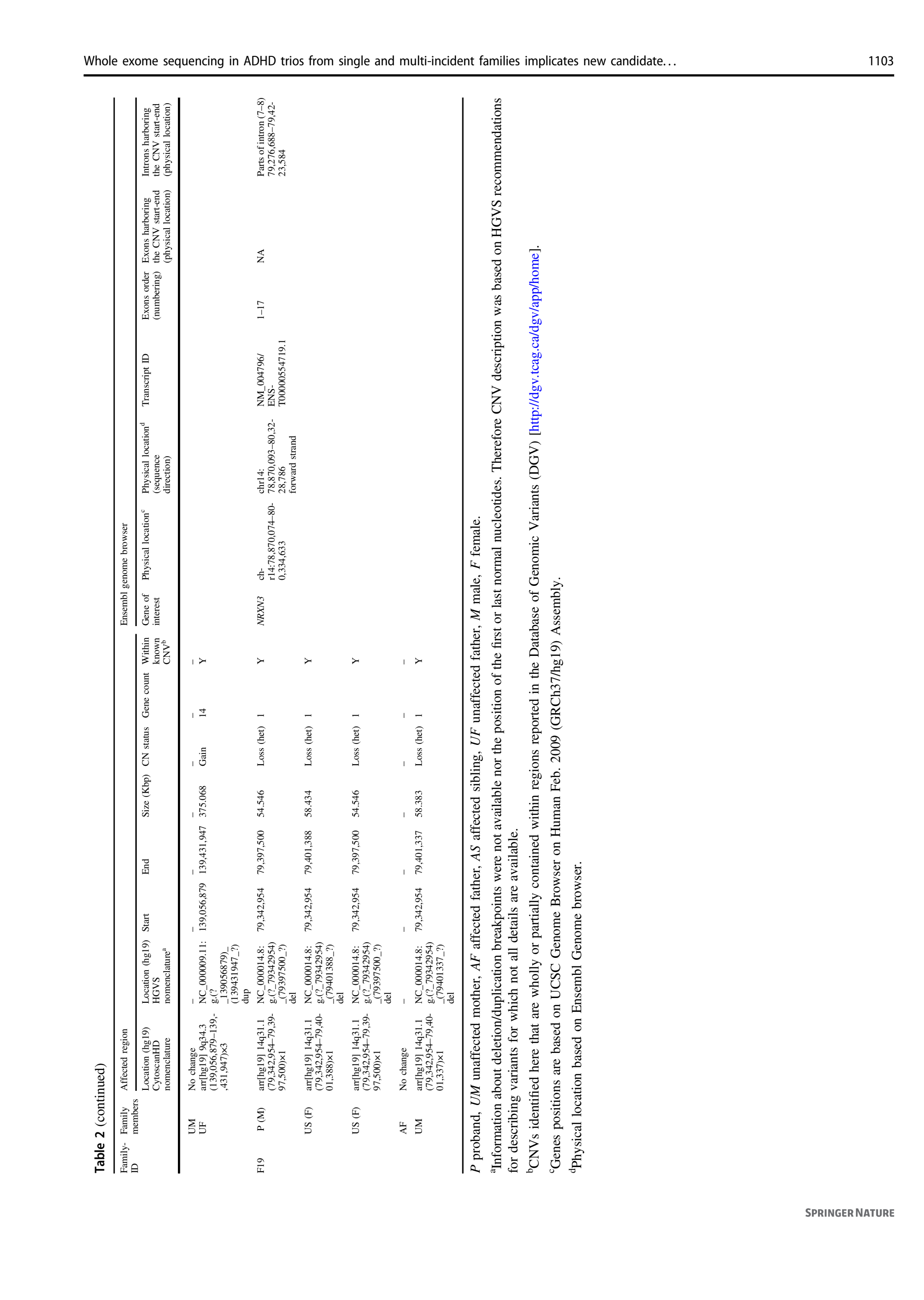


control checks except for F9 which was excluded from further analysis.

\section{Rare SNVs detected by WES}

By interrogating all protein coding regions covered by the employed kit, we have identified and validated a total of 32 unique rare variants in 31 different genes. Of which, 2 were de novo and 30 were inherited mainly being missense changes. Interestingly, 5 of the identified genes (NEK4, NLE1, PSRC1, PTP4A3, and TMEM183A) have not been previously described in any human disorder. The biological function of 10/31 genes is yet to be determined, while others participate in similar biological themes. For instance, signal transduction (THBS4, PLCB2, GNB4, PTCH1), chromatin modeling or DNA damage response (NEK4, ACIN1, PSRC1, TLK2, SPAG5), gene transcription regulation (KMT2A and ZFHX3), and vesicle transport (NBAS, KIF21A, PCLO) (Table S5).

Positive brain expression was reported for all the identified genes, also information on the developmental and regional differential expression was obtained from HBT (Table S6). Information was available for $26 / 32$ identified genes, 12 of which were expressed throughout the entire life span, 13 were under temporal and/or spatial control, and 1 gene (ACCS) had a negative expression in the brain. NLEl is the only gene that appears to be developmentally regulated across all included brain regions. Based on the HBT database, its expression is restricted to a limited developmental time frame (embryonic_late mid fetal development) [26].

The absence of a universal candidate gene/variant for neurodevelopmental disorders like ASD and ADHD is consistent with the increasingly accepted view that heterogeneous set of genetic variants, displaying incomplete penetrance, can shape risk to the disorder $[32,33]$. In light of this, we considered a model in which a combination of genetic variants forming a "constellation" may influence risk. Therefore, we have reported all SNVs that have survived our filtering criteria regardless of their co-segregation profile (Table 3). As expected, most of the validated variants exhibited reduced penetrance being either present in normally developing siblings or absent in the affected ones. One exception was LRRK1 LoF variant (c.2687-2A>G) that was detected in a homozygous state in three out the four affected individuals in F1, while the remaining (screened) family members including the 4th affected sibling were all heterozygous carriers for this change. These variants are an example of variation in penetrance and phenotypic expressivity, a phenomenon that is not uncommon in neurodevelopmental disorders.

Almost half of the genes identified herein (15/31) have a mouse model with documented behavioral or neurological phenotype (Table S7). Among these genes SLC9A9 was the only one with established mouse models of a neurodevelopmental disorder (ASD). This gene encodes a membrane protein localized in the late recycling endosomes, which are involved in the trafficking of neurotransmitters receptors and transporters. It is worth mentioning that we have screened all the genes listed in Table S1 for variants fulfilling our selection criteria to ensure that our pipeline had not missed any known causal or candidate variants in these genes. No candidate variants were flagged except the one that we have previously detected in SLC9A9. This gene was the only one overlapping with (IMAGE) [21].

\section{Biological pathways overrepresented in this study}

In order to investigate which biological processes might be affected by the variants identified herein, we utilized two web-based bioinformatics tools (WebGestalt and Reactome). A list of diverse functional categories were flagged as statistically overrepresented within our final set of genes with validated variants (Table S8). By querying KEGG and GO databases, WebGestalt was able to identify the top ten functional categories under "pathway", "biological process", "cellular component" and "molecular function" that are significantly enriched in our gene list. Various biological themes were highlighted including "Insulin secretion", "Glutamatergic, cholinergic, serotonergic, dopaminergic synapses", "Circadian rhythm", "Cell division", "Pyruvate dehydrogenase complex", and "Cytoskeleton organization". In addition, Reactome identified the 25 most significant pathways, examples of which include; "Regulation of insulin secretion", "Presynaptic function of Kainate receptors", and " $\mathrm{Ca}^{2+}$ pathway". Interestingly, among all the enriched functional categories "Insulin secretion" was identified as a recurrent theme.

\section{Discussion}

The role of rare SNVs has been much less investigated in ADHD, than in ASD, with no more than a handful of studies in which NGS was applied. These studies could be grouped into hypothesis-driven [14, 15], hypothesis- free [17-19] or a mix of both [16]. In the hypothesis-driven approach, NGS analysis was restricted to either a predefined set of ADHD candidate risk genes (IMAGE) [14], or gene sets curated from previous association studies (candidate genes/GWAS) after the application of specific criteria [15], or to a limited number of genes linked to ASD and intellectual disability (26 genes) [16]. With respect to sample size, all hypothesis-free studies were performed in a small number of cases/discovery-cohort (comprising 2-30 affected individuals), whereas larger number of samples (117-152 affected individuals) were included in the 


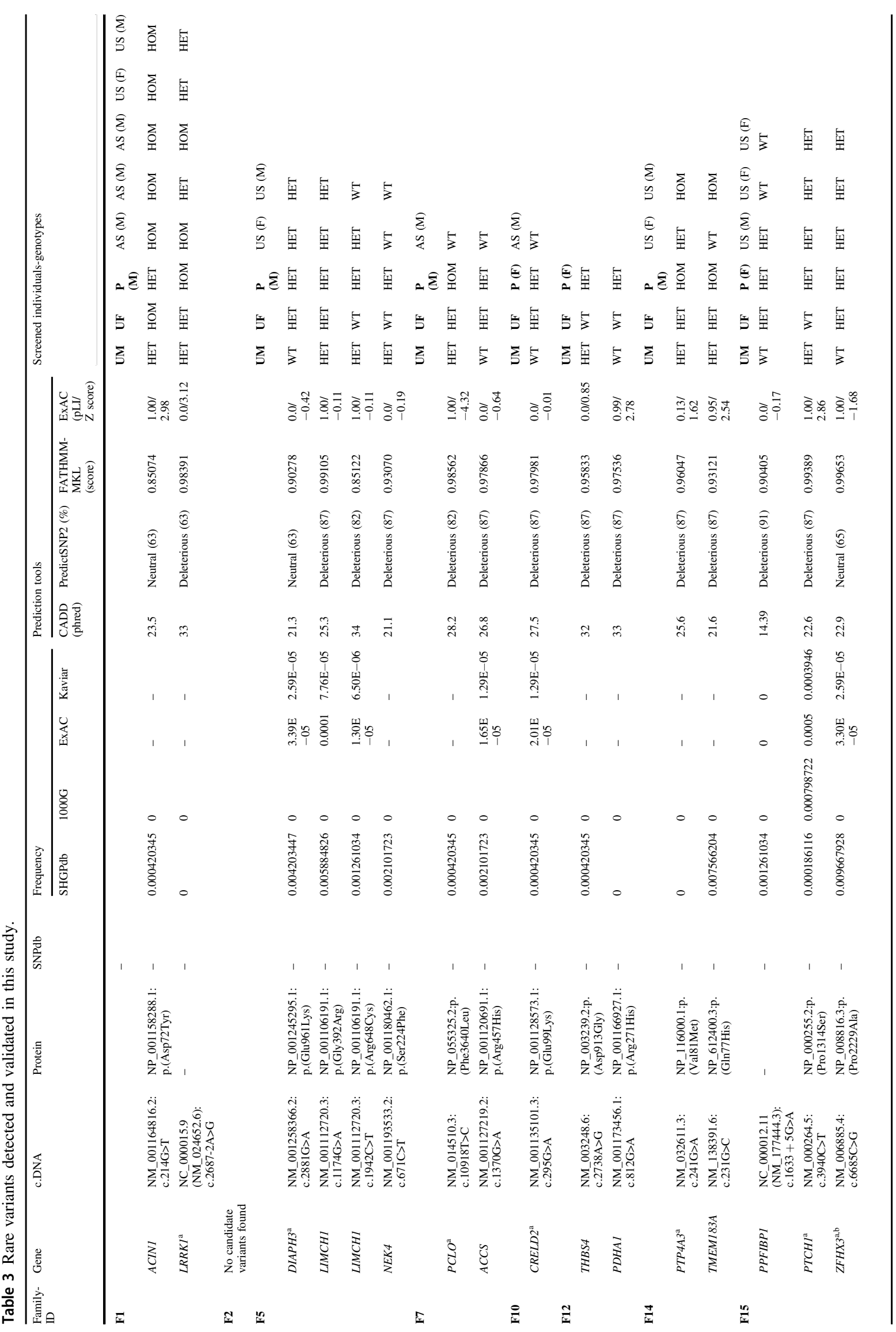




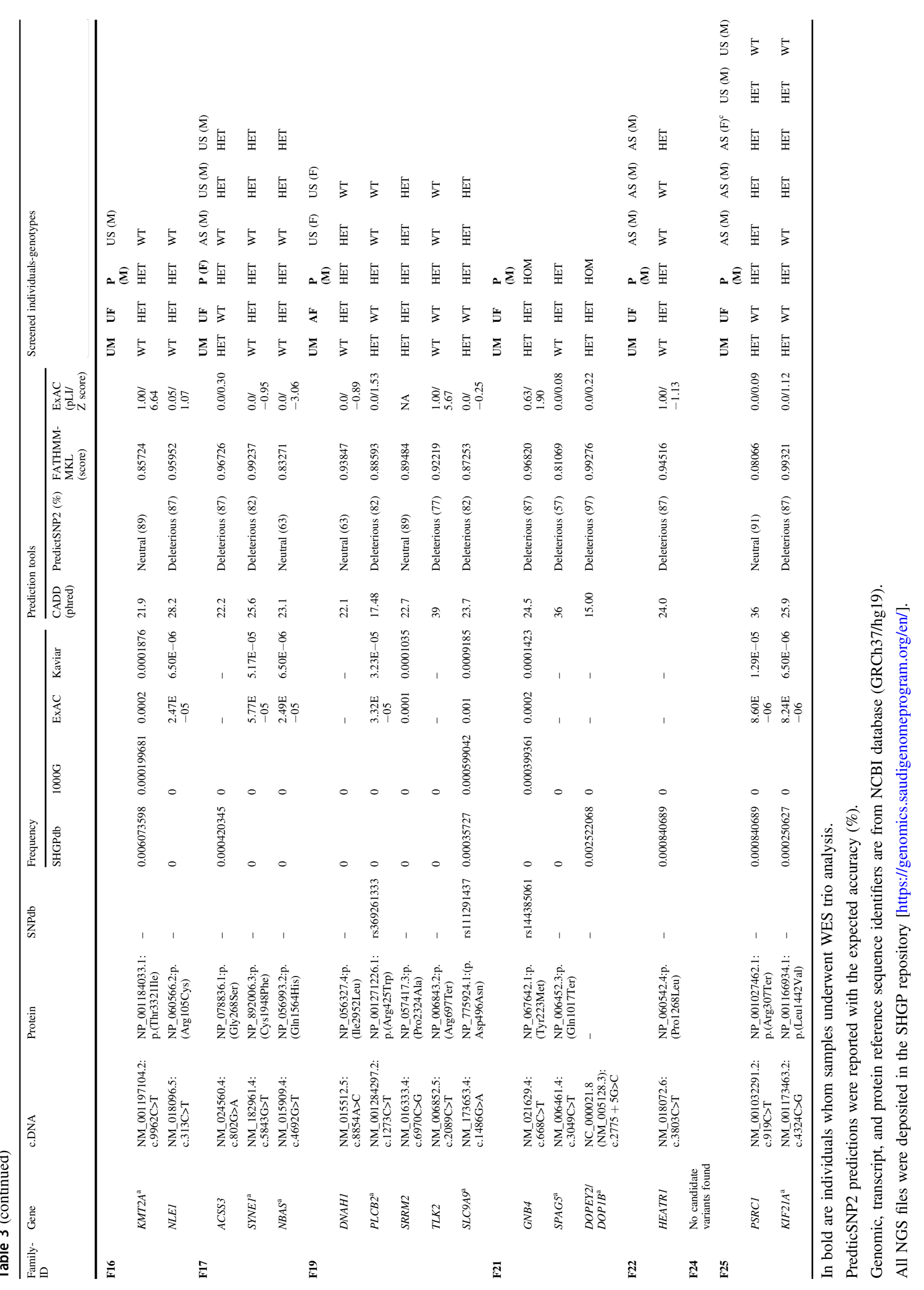




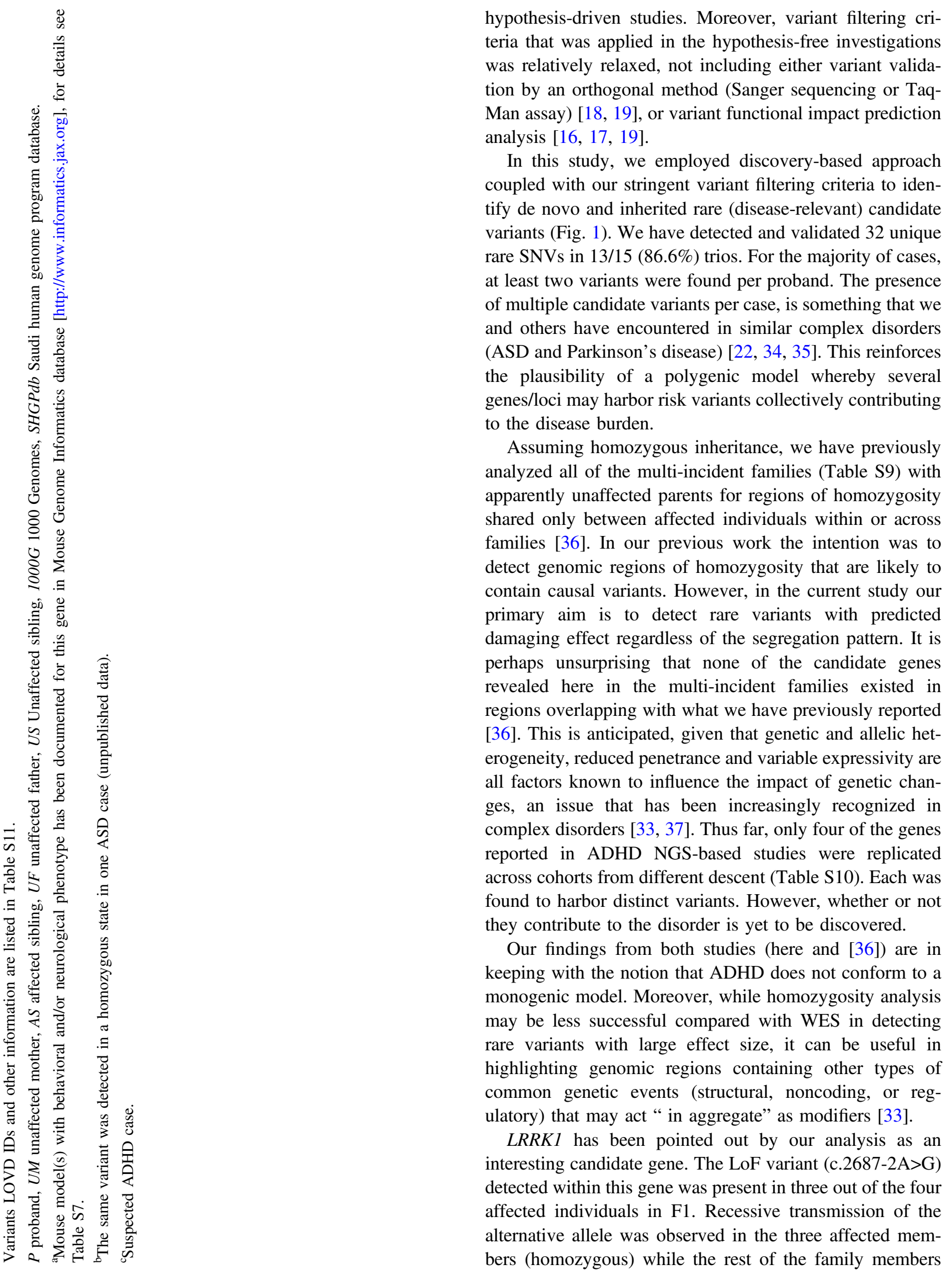


(4th affected sibling along with the parents and the healthy siblings) were all heterozygous carriers. Under the assumption of a compound heterozygous transmission, we revisited the data generated from the CytoScan ${ }^{\mathrm{TM}} \mathrm{HD}$ platform for any CNVs affecting $L R R K 1$ in the 4th affected individual (with a heterozygous genotype). However, no copy number changes were detected in this gene. In spite of the reduced penetrance of this variant, it is the only one in this study that was present in more than two affected individuals (same allele and genotype) which was not shared with the unaffected family members. This variant affects a splice-acceptor site located between intron 19 and exon 20 (exons are numbered from 1 to 34 based on NM_024652/ ENST00000388948.3, GRCh37 genome build) which maps onto the ROC-COR bidomain of the protein [38]. However, assessment of its consequence on gene splicing pattern was not possible here due to the unavailability of RNA samples.

Thus far, osteosclerotic metaphyseal dysplasia, which is a rare form of skeletal dysplasia, is the only human condition thought to be caused by variants in LRRK1 [39, 40]. Defective or depleted $L R R K 1$ has been shown to disrupt osteoclast normal function [39, 41]. Unfortunately, we could not determine whether the individuals homozygous for $L R R K 1$ variant had any form of bone abnormality due to the unavailability of relevant clinical and radiographic data.

Besides its role in bone homeostasis [41], LRRK1 have been shown to play a role in Grb2-mediated EGFR endocytic trafficking [42]. EGFR signaling influences many cellular processes including proliferation and migration of neural progenitor cells $[43,44]$ and more recently has been identified as an essential regulator of axon branching in the developing Drosophila brain [45]. Proper axonal branching is crucial for the formation of functional neural circuits; disruption of which may underlie brain circuitry aberrations that have been documented in individuals with ASD and ADHD [46, 47]. However, whether EGFR signaling regulates axonal branch formation in the mammalian brain or not, is yet to be determined.

Of the biological themes revealed by our enrichment analysis, at least five categories overlapped with prior findings "Glutamatergic synapse" (40 genes reported in ADHDgene database), "Dopaminergic synapse" (20 genes reported in ADHDgene database), "Serotonergic synapse", "Ca ${ }^{2+}$ signaling" (40 genes reported in ADHDgene database), and "Cytoskeleton organization". These biological processes are central to brain function therefore it is unsurprising to find that they have been repeatedly described in the literature [19, 48, 49]. Moreover, functional categories pertaining to the cytoskeleton comprised the largest number of genes (7/31) followed by "Regulation of insulin secretion" (14 genes reported in ADHDgene database) (3/31). Interestingly, insulin signaling may indirectly be involved in normal brain function through its known role in regulating the activity of both excitatory and inhibitory synapses in addition to influencing the brain's structural plasticity [50].

Small sample size is a limitation common to most of the published hypothesis-free ADHD genetic studies (including the present one) that can undermine the reliability of the produced findings. In fields such as human genetics of complex disorders, large-scale collaborative consortia are considered the gold standard approach for improving power and obtaining reliable and more generalizable results. However, extensive genetic and clinical heterogeneity not only across different cohorts/populations but also within families should be taken into consideration when this approach is employed. Another limitation of our study is that only the coding regions were interrogated for rare candidate variants, while variants existing outside our WES coverage area or those located in noncoding or regulatory regions were not possible to detect. In addition, the allele frequency of the CNVs identified here could not be determined due to the absence of data from matching normal controls.

In conclusion, our workflow revealed 32 unique rare variants in 31 different candidate genes most of which have not been previously described in ADHD. Even though our variant prioritization method is restricted to those with predicted deleterious effect, further functional and cellular analysis is essential to confirm their true biological consequences. The concept of a single causative variant being sufficient on its own to drive complex disorders like ADHD is no longer viable. Instead, an accumulating body of evidence indicates that multiple rare and common variants collectively contribute to the susceptibility to such disorders.

Acknowledgements NGS wet- and dry-lab procedures were all performed by the Saudi Human Genome Program (SHGP) at King Abdulaziz City for Science and Technology (KACST) and King Faisal Specialist Hospital and Research Centre (KFSHRC). We also would like to thank sequencing and genotyping core facilities in the department of Genetics at KFSHRC for performing Sanger sequencing and Cytoscan runs. This work was carried out as part of an approved project by KFSHRC (RAC\# 2120001).

Funding This study was funded by King Abdulaziz City for Science and Technology (KACST) [http://kacst.edu.sa/] grant no. 12BIO2346-20. NAA is the recipient of this grant. Funding body was not involved in any stage of the study and had no role in the design of the study or collection, analysis and interpretation of data or in writing the manuscript. This study was funded by King Abdulaziz City for Science and Technology (KACST) [https://www.kacst.edu.sa/eng/Pa ges/default.aspx] grant no. 12-BIO2346-20.

\section{Compliance with ethical standards}

Conflict of interest The authors declare that they have no conflict of interest.

Publisher's note Springer Nature remains neutral with regard to jurisdictional claims in published maps and institutional affiliations. 
Open Access This article is licensed under a Creative Commons Attribution 4.0 International License, which permits use, sharing, adaptation, distribution and reproduction in any medium or format, as long as you give appropriate credit to the original author(s) and the source, provide a link to the Creative Commons license, and indicate if changes were made. The images or other third party material in this article are included in the article's Creative Commons license, unless indicated otherwise in a credit line to the material. If material is not included in the article's Creative Commons license and your intended use is not permitted by statutory regulation or exceeds the permitted use, you will need to obtain permission directly from the copyright holder. To view a copy of this license, visit http://creativecommons. org/licenses/by/4.0/.

\section{References}

1. Faraone SV, Biederman J, Mick E. The age-dependent decline of attention deficit hyperactivity disorder: a meta-analysis of followup studies. Psychol Med. 2006;36:159-65.

2. Faraone SV, Larsson H. Genetics of attention deficit hyperactivity disorder. Mol Psychiatry. 2018;24:562-75.

3. Chou IJ, Kuo CF, Huang YS, Grainge MJ, Valdes AM, See LC, et al. Familial aggregation and heritability of schizophrenia and co-aggregation of psychiatric illnesses in affected families. Schizophr Bull. 2017;43:1070-8.

4. Cardno AG, Owen MJ. Genetic relationships between schizophrenia, bipolar disorder, and schizoaffective disorder. Schizophr Bull. 2014;40:504-15.

5. Thapar A, Cooper M, Eyre O, Langley K. What have we learnt about the causes of ADHD? J Child Psychol Psychiatry. 2013;54:3-16.

6. Hayman V, Fernandez TV. Genetic Insights Into ADHD Biology. Front Psychiatry. 2018;9:251.

7. Demontis D, Walters RK, Martin J, Mattheisen M, Als TD, Agerbo E, et al. Discovery of the first genome-wide significant risk loci for attention deficit/hyperactivity disorder. Nat Genet. 2019;51:63-75.

8. Fadista J, Manning AK, Florez JC, Groop L. The (in)famous GWAS P-value threshold revisited and updated for low-frequency variants. Eur J Hum Genet. 2016;24:1202-5.

9. Du Rietz E, Coleman J, Glanville K, Choi SW, O'Reilly PF, Kuntsi J. Association of polygenic risk for Attention-deficit/ hyperactivity Disorder with co-occurring traits and disorders. Biol Psychiatry Cogn Neurosci Neuroimaging. 2018;3:635-43.

10. Anttila V, Bulik-Sullivan B, Finucane HK, Walters RK, Bras J, Duncan L, et al. Analysis of shared heritability in common disorders of the brain. Science. 2018;360. pii: eaap8757.

11. Williams NM, Franke B, Mick E, Anney RJ, Freitag CM, Gill M, et al. Genome-wide analysis of copy number variants in attention deficit hyperactivity disorder: the role of rare variants and duplications at 15q13.3. Am J Psychiatry. 2012;169:195-204.

12. Williams NM, Zaharieva I, Martin A, Langley K, Mantripragada $\mathrm{K}$, Fossdal R, et al. Rare chromosomal deletions and duplications in attention-deficit hyperactivity disorder: a genome-wide analysis. Lancet. 2010;376:1401-8.

13. Hawi Z, Cummins TD, Tong J, Johnson B, Lau R, Samarrai W, et al. The molecular genetic architecture of attention deficit hyperactivity disorder. Mol Psychiatry. 2015;20:289-97.

14. Demontis D, Lescai F, Borglum A, Glerup S, Ostergaard SD, Mors $\mathrm{O}$, et al. Whole-exome sequencing reveals increased burden of rare functional and disruptive variants in candidate risk genes in individuals with persistent Attention-deficit/hyperactivity Disorder. J Am Acad Child Adolesc Psychiatry. 2016;55:521-3.

15. Hawi Z, Cummins TD, Tong J, Arcos-Burgos M, Zhao Q, Matthews $\mathrm{N}$, et al. Rare DNA variants in the brain-derived neurotrophic factor gene increase risk for attention-deficit hyperactivity disorder: a next-generation sequencing study. Mol Psychiatry. 2017;22:580-4.

16. Kim DS, Burt AA, Ranchalis JE, Wilmot B, Smith JD, Patterson $\mathrm{KE}$, et al. Sequencing of sporadic attention-deficit hyperactivity disorder (ADHD) identifies novel and potentially pathogenic de novo variants and excludes overlap with genes associated with autism spectrum disorder. Am J Med Genet B Neuropsychiatr Genet. 2017;174:381-9.

17. Lyon GJ, Jiang T, Van Wijk R, Wang W, Bodily PM, Xing J, et al. Exome sequencing and unrelated findings in the context of complex disease research: ethical and clinical implications. Discov Med. 2011;12:41-55.

18. Corominas J, Klein M, Zayats T, Rivero O, Ziegler GC, Pauper $\mathrm{M}$, et al. Identification of ADHD risk genes in extended pedigrees by combining linkage analysis and whole-exome sequencing. Mol Psychiatry. 2018. https://doi.org/10.1038/s41380-018-0210-6.

19. Lima Lde A, Feio-dos-Santos AC, Belangero SI, Gadelha A, Bressan RA, Salum GA, et al. An integrative approach to investigate the respective roles of single-nucleotide variants and copynumber variants in Attention-Deficit/Hyperactivity Disorder. Sci Rep. 2016;6:22851.

20. Zhang L, Chang S, Li Z, Zhang K, Du Y, Ott J, et al. ADHDgene: a genetic database for attention deficit hyperactivity disorder. Nucleic Acids Res. 2012;40:D1003-9.

21. Brookes K, Xu X, Chen W, Zhou K, Neale B, Lowe N, et al. The analysis of 51 genes in DSM-IV combined type attention deficit hyperactivity disorder: association signals in DRD4, DAT1 and 16 other genes. Mol Psychiatry. 2006;11:934-53.

22. Al-Mubarak B, Abouelhoda M, Omar A, AlDhalaan H, Aldosari $\mathrm{M}$, Nester $\mathrm{M}$, et al. Whole exome sequencing reveals inherited and de novo variants in autism spectrum disorder: a trio study from Saudi families. Sci Rep. 2017;7:5679.

23. Battle A, Brown CD, Engelhardt BE, Montgomery SB. Genetic effects on gene expression across human tissues. Nature. 2017;550:204-13.

24. Wu C, Orozco C, Boyer J, Leglise M, Goodale J, Batalov S, et al. BioGPS: an extensible and customizable portal for querying and organizing gene annotation resources. Genome Biol. 2009;10:R130.

25. Krizman DB, Wagner L, Lash A, Strausberg RL, Emmert-Buck MR. The Cancer Genome Anatomy Project: EST sequencing and the genetics of cancer progression. Neoplasia. 1999;1:101-6.

26. Kang HJ, Kawasawa YI, Cheng F, Zhu Y, Xu X, Li M, et al. Spatio-temporal transcriptome of the human brain. Nature. 2011;478:483.

27. Adzhubei I, Jordan DM, Sunyaev SR. Predicting functional effect of human missense mutations using PolyPhen-2. Curr Protoc Hum Genet. 2013. https://doi.org/10.1002/0471142905. hg0720s76.

28. Bendl J, Musil M, Stourac J, Zendulka J, Damborsky J, Brezovsky J. PredictSNP2: a unified platform for accurately evaluating SNP effects by exploiting the different characteristics of variants in distinct genomic regions. PLoS Comput Biol. 2016;12:e1004962.

29. Kircher M, Witten DM, Jain P, O'Roak BJ, Cooper GM, Shendure J. A general framework for estimating the relative pathogenicity of human genetic variants. Nat Genet. 2014;46:310-5.

30. Vaags AK, Lionel AC, Sato D, Goodenberger M, Stein QP, Curran S, et al. Rare deletions at the neurexin 3 locus in autism spectrum disorder. Am J Hum Genet. 2012;90:133-41.

31. Wang J, Gong J, Li L, Chen Y, Liu L, Gu H, et al. Neurexin gene family variants as risk factors for autism spectrum disorder. Autism Res. 2018;11:37-43.

32. Betancur C. Etiological heterogeneity in autism spectrum disorders: more than 100 genetic and genomic disorders and still counting. Brain Res. 2011;1380:42-77.

33. Vorstman JA, Ophoff RA. Genetic causes of developmental disorders. Curr Opin Neurol. 2013;26:128-36. 
34. Farlow JL, Robak LA, Hetrick K, Bowling K, Boerwinkle E, Coban-Akdemir ZH, et al. Whole-exome sequencing in familial Parkinson disease. JAMA Neurol. 2016;73:68-75.

35. Yemni EA, Monies D, Alkhairallah T, Bohlega S, Abouelhoda M, Magrashi A, et al. Integrated analysis of whole exome sequencing and copy number evaluation in Parkinson's disease. Sci Rep. 2019;9:3344.

36. Shinwari JMA, Al Yemni EAA, Alnaemi FM, Abebe D, AlAbdulaziz BS, Al Mubarak BR, et al. Analysis of shared homozygosity regions in Saudi siblings with attention deficit hyperactivity disorder. Psychiatr Genet. 2017;27:131-8.

37. De Rubeis S, Buxbaum JD. Genetics and genomics of autism spectrum disorder: embracing complexity. Hum Mol Genet. 2015;24:R24-31.

38. Civiero L, Vancraenenbroeck R, Belluzzi E, Beilina A, Lobbestael E, Reyniers L, et al. Biochemical characterization of highly purified leucine-rich repeat kinases 1 and 2 demonstrates formation of homodimers. PLoS ONE. 2012;7:e43472.

39. Guo L, Girisha KM, Iida A, Hebbar M, Shukla A, Shah H, et al. Identification of a novel LRRK1 mutation in a family with osteosclerotic metaphyseal dysplasia. J Hum Genet. 2017;62:437-41.

40. Iida A, Xing W, Docx MK, Nakashima T, Wang Z, Kimizuka M, et al. Identification of biallelic LRRK1 mutations in osteosclerotic metaphyseal dysplasia and evidence for locus heterogeneity. J Med Genet. 2016;53:568-74.

41. Xing WR, Goodluck H, Zeng C, Mohan S. Role and mechanism of action of leucine-rich repeat kinase 1 in bone. Bone Res. 2017;5:17003.

42. Hanafusa $H$, Ishikawa $K$, Kedashiro S, Saigo T, Iemura S, Natsume $\mathrm{T}$, et al. Leucine-rich repeat kinase LRRK1 regulates endosomal trafficking of the EGF receptor. Nat Commun. 2011;2:158.
43. Kornblum HI, Yanni DS, Easterday MC, Seroogy KB. Expression of the EGF receptor family members ErbB2, ErbB3, and ErbB4 in germinal zones of the developing brain and in neurosphere cultures containing CNS stem cells. Dev Neurosci. 2000;22:16-24.

44. Lillien L, Raphael H. BMP and FGF regulate the development of EGF-responsive neural progenitor cells. Development. 2000;127:4993-5005.

45. Zschatzsch M, Oliva C, Langen M, De Geest N, Ozel MN, Williamson WR, et al. Regulation of branching dynamics by axonintrinsic asymmetries in tyrosine kinase receptor signaling. Elife. 2014;3:e1699.

46. Chen H, Wang J, Uddin LQ, Wang X, Guo X, Lu F, et al. Aberrant functional connectivity of neural circuits associated with social and sensorimotor deficits in young children with autism spectrum disorder. Autism Res. 2018;11:1643-52.

47. Gehricke JG, Kruggel F, Thampipop T, Alejo SD, Tatos E, Fallon $\mathrm{J}$, et al. The brain anatomy of attention-deficit/hyperactivity disorder in young adults - a magnetic resonance imaging study. PLoS ONE. 2017;12:e175433.

48. Ohgi Y, Futamura T, Hashimoto K. Glutamate signaling in synaptogenesis and NMDA receptors as potential therapeutic targets for psychiatric disorders. Curr Mol Med. 2015;15:206-21.

49. Stanford SC. Psychostimulants, antidepressants and neurokinin-1 receptor antagonists ('motor disinhibitors') have overlapping, but distinct, effects on monoamine transmission: the involvement of L-type $\mathrm{Ca} 2+$ channels and implications for the treatment of ADHD. Neuropharmacology. 2014;87:9-18.

50. Kleinridders A, Ferris HA, Cai W, Kahn CR. Insulin action in brain regulates systemic metabolism and brain function. Diabetes. 2014;63:2232-43. 